\title{
A Retrospective, Observational Study to Determine the Patient and Tumor Characteristics of HER2-Positive Breast Cancer Patients Treated at Five Main Public Cancer Centers in Malaysia
}

\author{
Ros Suzanna Ahmad Bustamam¹ Yu Kong Leong ${ }^{2}$ \\ Yew-Teik Cheong ${ }^{5}$ Goh Kenny ${ }^{6}$ \\ ${ }^{1}$ Division of Clinical Oncology, Department of Radiotherapy and \\ Oncology, Hospital Kuala Lumpur, Kuala Lumpur, Malaysia \\ ${ }^{2}$ Division of Clinical Oncology, Hospital Umum Sarawak, Kuching, \\ Sarawak, Malaysia \\ ${ }^{3}$ Division of Clinical Oncology, Hospital Wanita Kanak-Kanak Sabah, \\ Kota Kinabalu, Sabah, Malaysia \\ ${ }^{4}$ Division of Clinical Oncology, Institut Kanser Negara, Putrajaya, \\ Wilayah Persekutuan, Malaysia \\ ${ }^{5}$ Consultant Breast Surgeon, Hospital Pulau, Pinang, Malaysia \\ 6 Medical Affairs Roche (Malaysia) Sdn. Bhd., Kuala Lumpur, Malaysia
}

Flora Li Tze Chong ${ }^{3}$ Florence Wong Yoke Fui ${ }^{4}$

Asian J Oncol 2020;6:10-19

\begin{abstract}
Address for correspondence Ros Suzanna Ahmad Bustamam, MCO (UM), MBBCh, BAO, LRCP\&SI, MCO (UM), MBBCh, BAO, LRCP\&SI, Department of Radiotherapy and Oncology, Hospital Kuala Lumpur, Jalan Pahang, Wilayah Persekutuan, Kuala Lumpur 50586, Malaysia (e-mail: rossuzanna@gmail.com).
\end{abstract}

\begin{abstract}
Keywords

- HER2-positive breast cancer

- Malaysia

- prognosis

Introduction Human epidermal growth factor receptor 2 (HER2) overexpressing breast cancer is a high-risk subtype with poor prognosis. The incidence of HER2 expressing tumors is high in Malaysia; however, there is limited information on the characteristics of these tumors. Therefore, we sought to collect the patient and tumor characteristics of HER2+ breast cancer cases at five centers in Malaysia.

Patients and Methods A retrospective review was conducted of the data from charts of patients diagnosed with HER2+ breast cancer between January 2014 and December 2015 at Hospital Kuala Lumpur, Institute Kanser Negara, Hospital Pulau Pinang, Hospital Besar Sarawak, and Hospital Likas in Malaysia.

Results Of the 1,519 screened patient' charts, 396 were included for the analysis. The average age of HER2+ breast cancer cases at diagnosis was 51.07 years. A high percentage of cases presented at an advanced stage (38.89 and 12.12\% with stage 3 and 4 , respectively). About $58.84 \%$ of patients were categorized as "high-risk," with one or more lymph node involvement. Close to half (47.98\%) of cases presented with T2 stage tumors, and infiltrating ductal carcinomas were reported in $85.35 \%$ of tumors. The most common immunohistochemical subtype was estrogen receptor (ER)+/progesterone receptor (PR)+/HER2+ (47.47\%), followed by ER-/PR-/HER2+ (37.12\%).

Conclusion HER2 overexpressing tumors represent an aggressive subtype in Malaysia with large tumor size, high tumor grade, and lymph node involvement. Early diagnosis and management of these tumors may help improve the survival rates. Future studies should help elucidate the treatment patterns and outcomes in HER2+ breast cancer patients in Malaysia.
\end{abstract}

DOI https://doi.org/

$10.1055 / \mathrm{s}-0040-1708108$

ISSN 2454-6798.
(C)2020 Spring Hope Cancer Foundation \& Young Oncologist Group of Asia
License terms

() (1) $\ominus \circledast$ 


\section{Introduction}

Breast cancer is the second most common cancer in the world, with an incidence rate of $11.9 \%$, accounting for $25 \%$ of all diagnosed cancer cases. The incidence and mortality rates have been consistently increasing in less-developed regions compared with developed regions of the world. This shift in the global distribution of breast cancer cases has positioned it as a major health concern in Asia and Southeast Asia. ${ }^{1}$ About a quarter of all breast cancer cases are diagnosed in the Asia-Pacific region; the highest rate is observed in Singapore (65 per 100,000 cases) followed by Brunei (48.6), the Philippines (47), Indonesia (40.3), Malaysia (38.7), Timor-Leste (32.6), Thailand (29.3), Vietnam (23), Myanmar (22.1), Cambodia (19.3), and Laos (19). ${ }^{2}$ Breast cancer-related mortality rate has also risen sharply in the female population of Southeast Asian region, with the highest rate reported in Malaysia (18.9 per 100,000 ), followed by the Philippines (17.8), Indonesia (16.6), Timor-Leste (16.4), Singapore (15.5), Myanmar (11.3), Brunei (11.3), Thailand (11.0), Vietnam (9.9), Laos (9.3), and Cambodia (9.3). ${ }^{2}$

In Malaysia, 32\% of cancer cases in women were diagnosed as breast cancer from 2007 to 2011. The lifetime risk of developing breast cancer has been noted to be highest among the Chinese ( 1 in 22) followed by Indian (1 in 24) and Malay ( 1 in 35 ) ethnicities in this region. About $57 \%$ of breast cancer cases are diagnosed as either stage I or II, and $43 \%$ as stages III and IV. ${ }^{3}$ A survey of breast cancer incidence within various regions of Malaysia has revealed that Penang tops the charts with 50 cases per 100,000 , followed by Putrajaya (42 cases) and Johor (39 cases). ${ }^{3}$ The percentage of breast cancer cases in Penang has increased from $14.5 \%$ in 2003 to $17.7 \%$ in $2008 .{ }^{4}$ In regional studies, several factors have been found to be associated with an increased risk of breast cancer, such as (1) having a positive family history of breast cancer, (2) obesity, (3) physical inactivity, (4) oral contraceptive use, and (5) shorter duration of breastfeeding. . $^{510}$

Despite the high incidence of breast cancer and prevailing risk factors, there are several unmet needs or challenges in the diagnosis and management of breast cancer in Malaysia that need to be addressed. One of the major challenges is the high percentage of cases being diagnosed at a late stage due to the delay in presentation and consultation. Health care system deficiencies such as delayed referral by clinicians, poor patient-practitioner relationship, miscommunication, lack of clarity about treatment, and loss of patient follow-up have exacerbated this problem. ${ }^{11}$ Another important factor contributing to delayed diagnosis is the lack of knowledge of the symptoms, bundled with fatalistic beliefs and uncertainty of treatment outcomes in the Malaysian female population. ${ }^{11}$ Social-cultural factors, such as lack of family support, excessive dependency on family to make critical decisions, and perception of losing femininity due to invasive surgical interventions, have deterred middle-aged Malaysian women from seeking timely breast cancer treatment. ${ }^{11}$ The existence of alternative therapies and the staunch belief that alternative medicine can cure every disease has further impaired the timely identification and management of breast cancer cases among all ethnic groups of Malaysia. ${ }^{11,12}$ All these factors have cumulatively increased the percentage of breast cancer cases being diagnosed at a late stage and lowered the overall survival rate. ${ }^{13}$ The survival rate has been found to be much lower among Malaysian women with breast cancer compared with their counterparts in Western countries. ${ }^{14}$

Breast cancer can be categorized into four different subtypes based on the gene profiling and immunophenotypic characteristics, each subtype differing from the other in clinical presentation, aggressiveness, treatment response, and prognosis: (1) luminal A (estrogen receptor [ER]+ and/or progesterone receptor $[\mathrm{PR}]+$, human epidermal growth factor receptor 2 [HER2]-) and luminal B (ER+ and/or PR+, HER2+), both characterized by high levels of ER and genes activated by ER transcription factors, and exhibit similar profiles to those of normal cells of the luminal epithelium of the mammary glands; (2) basal cell-like (BCL) (ER-, PR-, HER2-), also known as "triple negative," are negative for ER and PR with expression of genes usually in basal/myoepithelial cells of the breast; (3) HER2 overexpressing tumors (ER-, PR-, HER2+), characterized by amplification of the HER2/neu gene and genes associated with HER2 pathways; and (4) normal breast-like tumors. ${ }^{15,16}$ While the luminal A tumors have been found to be less aggressive with a good prognosis, the $\mathrm{BCL}$ type displays aggressive behavior, lack of response to endocrine therapy, mutations of BRCA1 gene, shorter overall survival, and poor prognosis. ${ }^{16}$

The HER2 overexpressing type accounts for 15 to $20 \%$ of breast tumors, and is aggressive, with poor prognosis, high recurrence rate, shortened disease-free periods, and decreased overall survival. ${ }^{17-20}$ The apparent reason for the poor prognosis of HER2 overexpressing tumors can be attributed to factors such as age, tumor size, tumor grade, and lymph node involvement. ${ }^{17}$ The highest HER2 positivity rate in breast cancer tumors in the Asian region has been observed in women below the age of 40 years (38.2\%). ${ }^{17}$ HER2 overexpression has been frequently observed in tumors with large mean size and is significantly associated with stage $3(43.3 \%)$ and stage 4 tumors (39.6\%). ${ }^{16-18} \mathrm{~A}$ high positive involvement of lymph node has also been reported in the HER2/neu subtypes. ${ }^{16}$ Furthermore, these tumors are overexpressed more in ER-breast cancers (41.6\%) compared with ER+ types (21.5\%). ${ }^{18}$ Due to all these factors, HER2 overexpressing tumors represent a high-risk subtype with poor prognosis.

An audit of 30,371 patients with breast cancer across 8 Asian regions revealed that the rate of incidence of the HER2+ type of breast cancer was higher in these regions compared with Western countries, the highest seen in Malaysia (51.6\%). ${ }^{17}$ A separate analysis of 1,963 breast cancer patient in Sarawak, Malaysia, reported the incidence of the HER2+ subtype to be dominant in the Malay ethnic group (23\%), compared with other ethnicities. ${ }^{21}$ In addition to the high prevalence of HER2+ breast cancer, one of the major unmet needs in the diagnosis of HER2+ breast cancer patients in Malaysia is the reduced probability of testing for HER2 status: Although HER2 testing is recommended for immunohistochemistry (IHC) $2+$ or $3+$ cases, ${ }^{22}$ high-risk breast cancer patients are less likely to be tested for HER2 status, as clinicians generally base their decision on testing the patient for HER2 status on the probable prognosis at the time of 
diagnosis. ${ }^{23}$ Another unmet need is the lack of an overall accurate picture of the presentation of HER2+ cases in Malaysia; this can be attributed to design limitations of the existing studies, which are mostly confined to single-center design. Through our study, we aimed to collect the patient and tumor characteristics of HER2+ breast cancer cases at multiple centers in Malaysia.

\section{Patients and Methods}

\section{Study Design}

This is a descriptive, observational study conducted using retrospective data from five centers within the Ministry of Health hospitals in Malaysia.

\section{Study Centers}

Following a feasibility assessment, nine centers were shortlisted and assessed for the number of available records from breast cancer patients. Only five centers with the highest number of records were selected; no other factors were considered during site selection. The centers selected for this study were Hospital Kuala Lumpur and Institute Kanser Negara from Kuala Lumpur, Hospital Pulau Pinang from Penang, Hospital Besar Sarawak from Kuching, and Hospital Likas from Kota Kinabalu.

\section{Study Population}

HER2-positive breast cancer patients, either in the early or metastatic stage diagnosed between January 1, 2014 and December 31, 2015, were included. Patients who received any anticancer treatment, including hormonal therapy, chemotherapy, and/or anti-HER2 treatment, were also considered. Patients with HER2-negative type breast cancer or any other concurrent primary cancer were excluded from the study.

\section{Data Source}

Data were retrieved from medical records and patient notes (paper and electronic formats) from the study centers. Clinical data analysts were tasked with reviewing data to check for logic, data consistency, spelling errors, clinically appropriate terms, and any other missing information. Quality control of data was performed at regular intervals to identify any errors and the same was shared with the data analyst.

\section{Study Variables}

The demographic variables collected included gender, age, and weight when diagnosed, date of diagnosis, method of initial diagnosis, method to determine HER status, ethnicity, state of residence, and family history of breast cancer or other cancers. The clinicopathological variables collected included the stage of cancer, tumor grade, tumor size, lymph node involvement, metastasis, tumor histology, and IHC subtype.

\section{Risk Stratification}

In our study, we considered patients to be at intermediate risk if they had no lymph node involvement and satisfied at least one of the following criteria: tumor size $>2 \mathrm{~cm}$, tumor grade $2 / 3$, age $<35$ years, or vessel invasion. Patients who had one or more lymph nodes involved were categorized as "high-risk." A similar risk stratification method has been proposed by the St Gallen consensus panel of experts and used in the prognostication of breast cancer patients in studies. ${ }^{24,25}$

\section{Statistical Analysis}

Descriptive statistics such as counts and percentages were used for categorical data, and means were calculated for continuous variables. Source data verification was performed for missing data, and subjects with missing data were not considered for analysis. A sensitivity analysis was not used in the study. Standard deviations and confidence intervals were not calculated, as this study did not test any hypothesis.

\section{Results}

A total of 1,519 patient charts were screened and, based on the inclusion/exclusion criteria, 408 patients were selected. About 12 patients were excluded during the study, resulting in 396 patients included for analysis.

\section{Demographic Data}

Of the 396 HER2+ patients included in the study, 394 (99.49\%) were female and $2(0.51 \%)$ were male. The average weight at diagnosis was recorded to be $60.10 \mathrm{~kg}$, and the average age at diagnosis was 51.07 years. The age-wise distribution of the patients has been shown in - Fig. 1. About $15.64 \%$ of patients were diagnosed with breast cancer at $<40$ years of age and

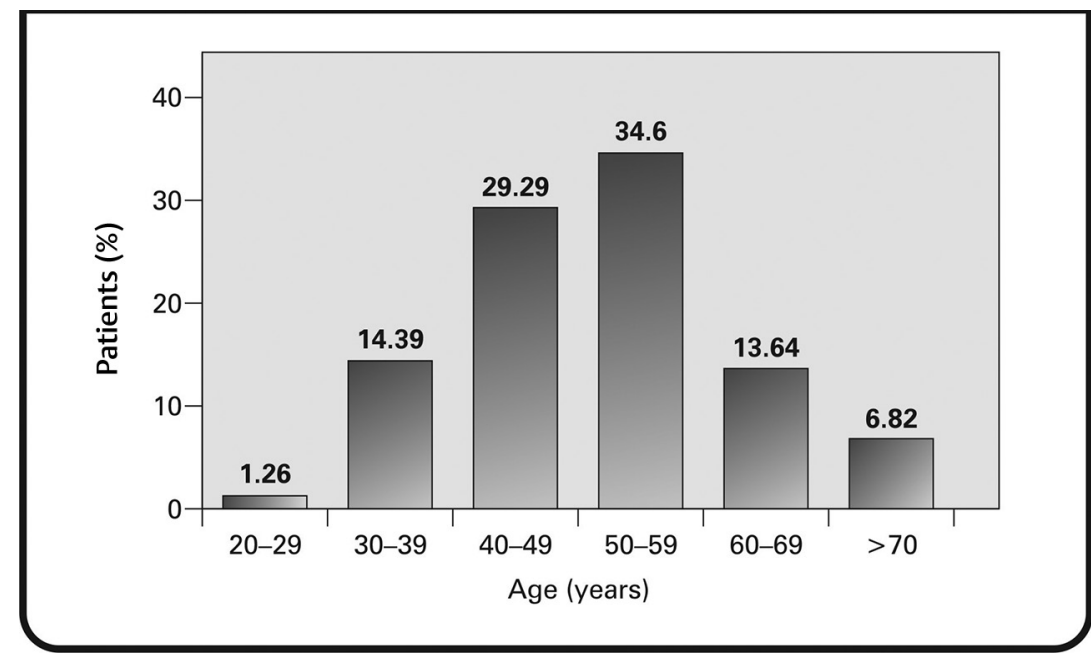

Fig. 1 Age at the time of diagnosis of human epidermal growth factor receptor $2+$ breast cancer. 
$44.94 \%$ of patients were diagnosed at $<50$ years of age. The most common ethnicity was found to be Chinese (40.66\%), followed by Malay (29.04\%) and Indian (7.32\%). Regional distribution data revealed that most patients came from Sarawak (26.26\%), Selangor (21.72\%), and Sabah (20.45\%) regions (-Fig. 2).

A family history of breast cancer was reported by $18.94 \%$ of patients, and a lack of breast cancer family history was reported by $58.08 \%$. A family history of other cancers was reported in $16.6 \%$, and $56.06 \%$ reported a lack of family history for other cancers.

For the initial diagnosis of breast cancer, methods such as physical examination, biopsy, mammogram, and ultrasound were used. Patients were stratified as intermediate or high-risk. Data were available for $93.18 \%$ of the patients, of whom $34.34 \%$ were categorized as intermediate-risk and 58.84\% as high-risk (-Fig. 3 ).

\section{Clinicopathology Data}

HER2 status was analyzed using IHC (97.73\% of patients) and/or in-situ hybridization (ISH) (92.68\% of patients). For tumors assessed via IHC, 21.96\% were 2+ (borderline), 76.23\% were 3+ (HER2+), and 1 tumor was 1+ (negative for HER2). For tumors assessed via ISH, $97.02 \%$ had results $>2.2$ (positive for HER2) and $1.63 \%$ had results between 1.8 and 2.2 (equivocal for HER2). One tumor had ISH results that were inconclusive $(<1.8)$, but upon consideration of IHC results, it was included as HER2+ (-Figs. 4A and B ).

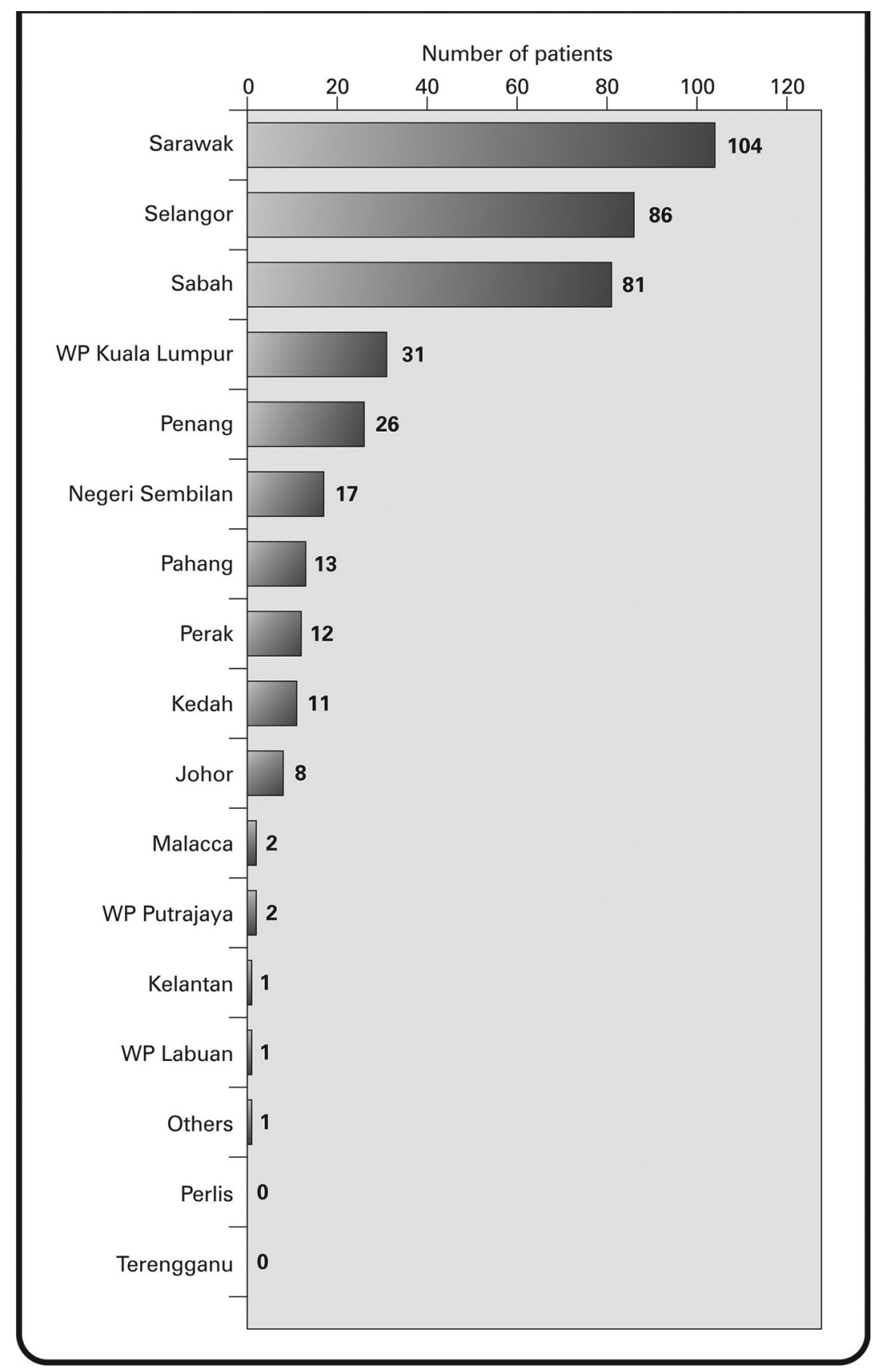

Fig. 2 Human epidermal growth factor receptor 2+ breast cancer patient's distribution, by state, in Malaysia. 


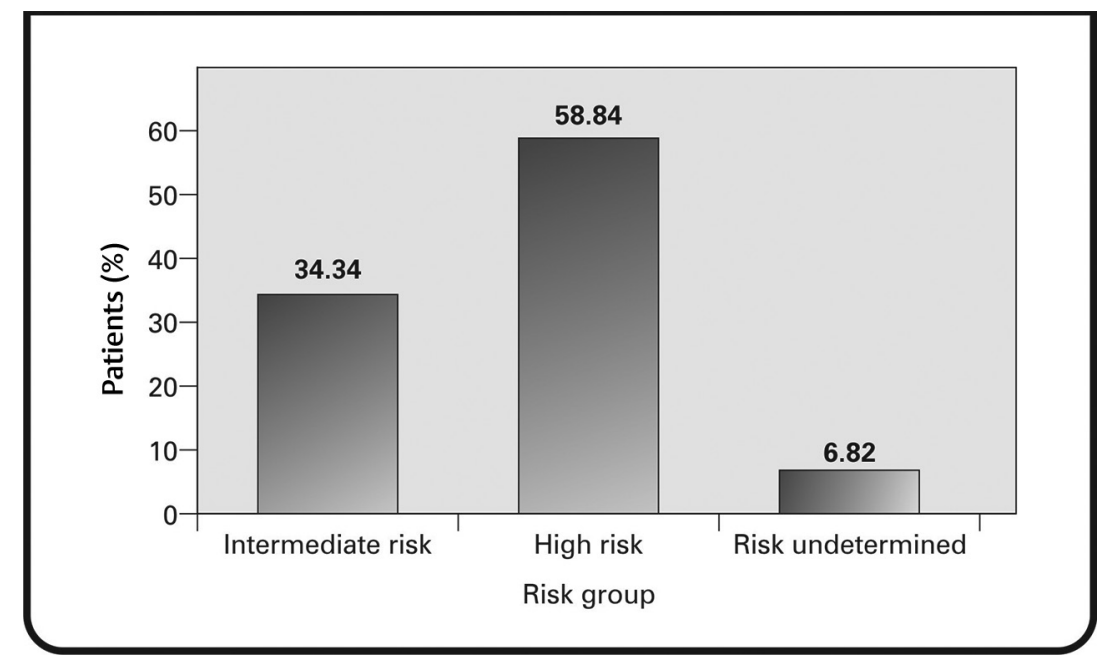

Fig. 3 Risk stratification of patients.

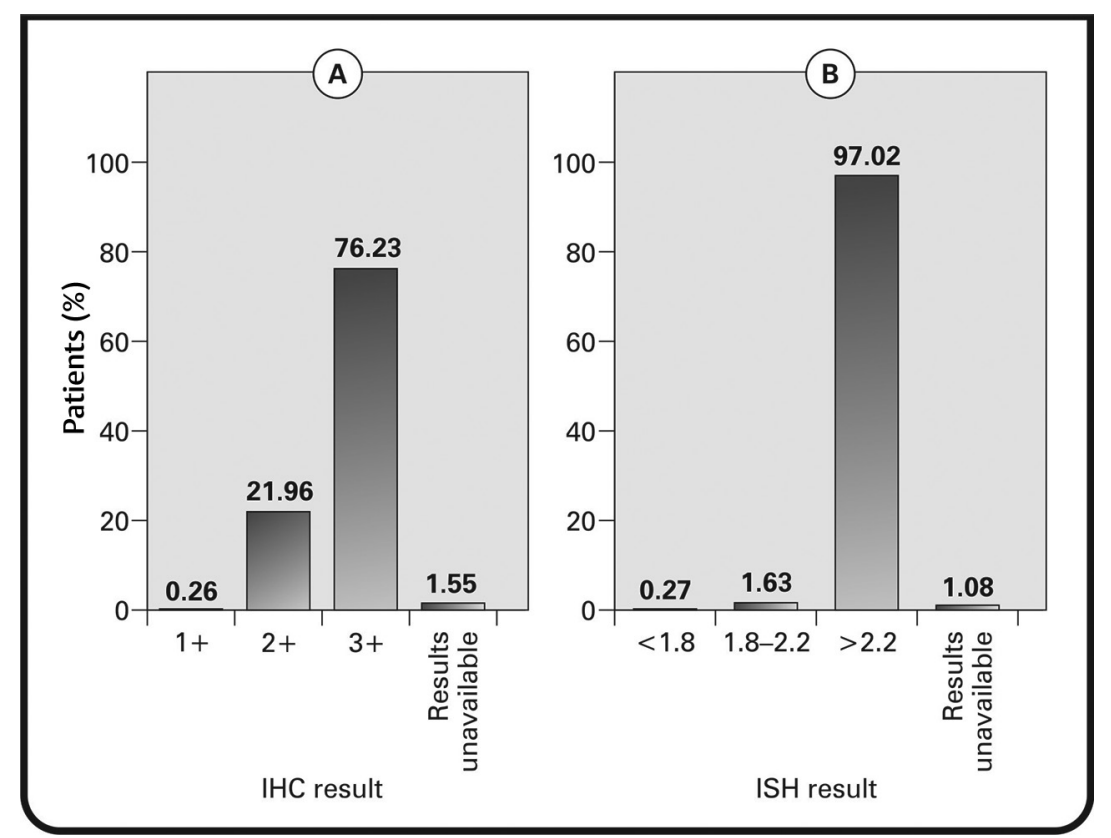

Fig. 4 (A) Immunohistochemistry (IHC) results in patients testing for human epidermal growth factor receptor 2+ (HER2+) status; (B) in-situ hybridization (ISH) results showing patients positive for HER2.

Cancer staging and grading were performed at diagnosis. It was found that $48.74 \%$ of the patients were at stage 1 or 2 ; $38.89 \%$ were at stage 3 , and $12.12 \%$ were at stage 4 (-Fig. 5). Tumor sizing was achieved using the tumor, node, metastasis (TNM) staging system. T1 tumors were found in $22.73 \%$ of patients, T2 in $47.98 \%$ of patients, T3 tumors in $15.40 \%$ of patients, and $\mathrm{T} 4$ tumors in $12.12 \%$ of patients (-Fig. 6). Lymph node involvement was observed in most patients. One to three positive nodes involvement were seen in $27.53 \%$ of patients, 4 to 10 positive nodes in $19.19 \%$ of patients, and more than 10 positive nodes in $12.12 \%$ of patients. Metastasis was observed in $12.37 \%$ of patients.

Infiltrating ductal carcinoma, not otherwise specified, was seen in $85.35 \%$ of patients. Other types of carcinoma were seen in $14.39 \%$ of the patients (-Fig. 7 ). The most common immunohistochemical subtype was $\mathrm{ER}+/ \mathrm{PR}+1$
HER+ (47.47\%), followed by ER-/PR-/HER2+ (37.12\%). The remainder of the tumors were ER+/PR-/HER2+ (13.89\%) and ER-/PR+/HER2+ (1.52\%) ( - Fig. 8).

\section{Discussion}

Breast cancer is a heterogeneous complex of diseases with a spectrum of many subtypes. The HER2+ breast cancer subtype accounts for 15 to $20 \%$ of breast cancer cases and is characterized by aggressive clinical behavior. ${ }^{26}$ The aim of our study was to collect the patient and tumor characteristics of HER2+ breast cancer patients in Malaysia.

In our analysis of 1,519 charts reviewed across five centers, 396 (26.06\%) patients were found to have HER2+ tumors. The most affected age group comprised individuals aged between 50 and 59 years (34.51\%), with a mean age of 


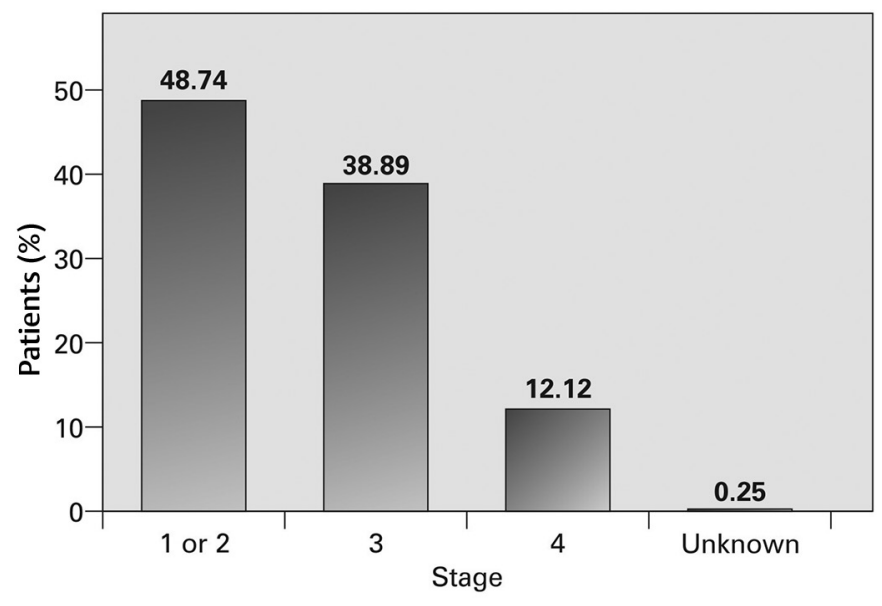

Fig. 5 Percentage of patients at different stages of breast cancer at diagnosis.

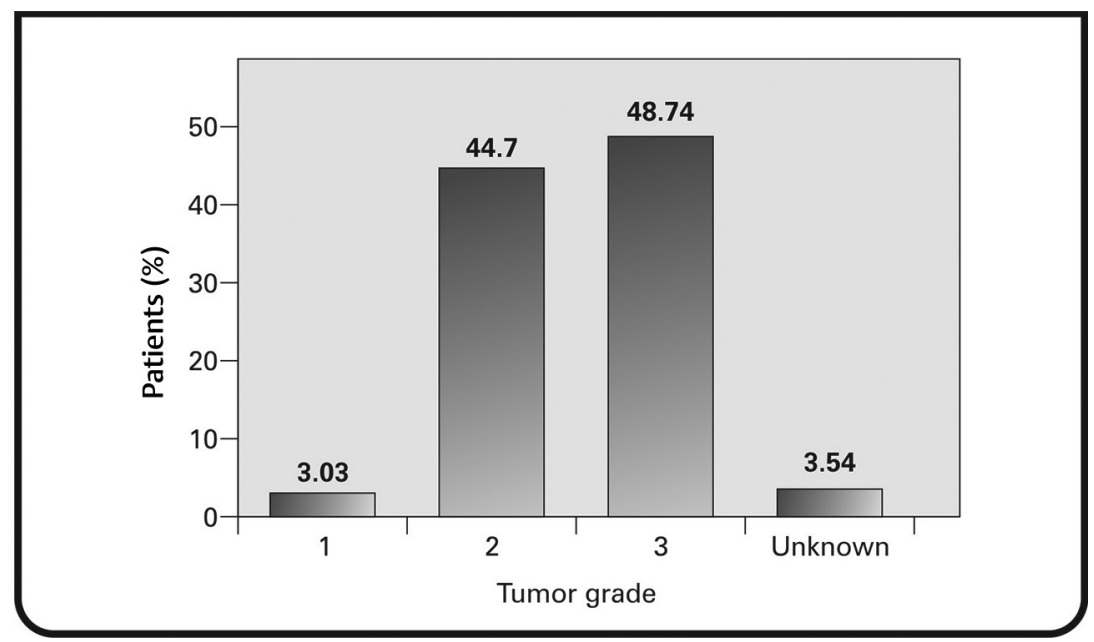

Fig. 6 Tumor grading based on TNM criteria. TNM, tumor, node, metastasis.

51.09 years. Our findings are consistent with those reported by Tan et al, where the median age of breast cancer patients with HER2 overexpressing tumors at a medical center in Malaysia was 53.3 years. ${ }^{27}$ Overall, the mean age at diagnosis of breast cancer in Asian countries, including Malaysia, has been noted to be low compared with Western countries. ${ }^{23,28}$ The plausible factors accounting for a younger mean age of presentation with breast cancer in Malaysia may be due to the younger demographic (median age: 26.1 years), and lower lifestyle-related risk factors (more children, low urbanization, and more breast feeding) in the current older population in Malaysia, resulting in a lower risk of postmenopausal breast cancer in this population. ${ }^{23}$

We observed in our study that HER2+ tumors were seen primarily in the women of Chinese ethnicity (40.66\%). This contrasts with findings from previous observations, wherein, the percentage of triple-positive HER2 cases was higher for the Malay (16\%) ethnicity, followed by the Chinese (11\%). ${ }^{21}$ Analysis of patient distribution in our study revealed that a majority of the patients were from Sarawak, followed by Selangor, Sabah, WP Kuala Lumpur, and Penang. Breast cancer has been identified as the commonest cancer in women of Sarawak state; the number of cancer cases has increased by 2.9-fold as per the data recorded in $2010 .{ }^{21}$ However, the distribution of HER2+ cancer cases in our study is not completely in line with the region-wise incidence of overall breast cancer cases reported in the National Cancer Registry report 2007 to 2011. Although a high incidence of breast cancer has been reported in all the cited regions in this report, the descending order of the incidence of breast cancer has been found to be $50,42.2,31.7,22.7$, and 19.7 per 100,000 population in Penang, WP Kuala Lumpur and Putrajaya, Selangor, Sarawak, and Sabah, respectively. ${ }^{3}$

We reviewed the literature to identify the relationship between family history and the occurrence of breast cancer. A family history of breast cancer is considered a major risk factor for breast cancer. A study conducted among 400,000 women aged 65 years and older reported that first-degree family history was associated with an increased risk of invasive breast cancer among all subgroups. ${ }^{29}$ It was observed that the 5 -year risk increased by $23 \%$ for women with a family history of breast cancer. ${ }^{29}$ Another study of 113,000 women 


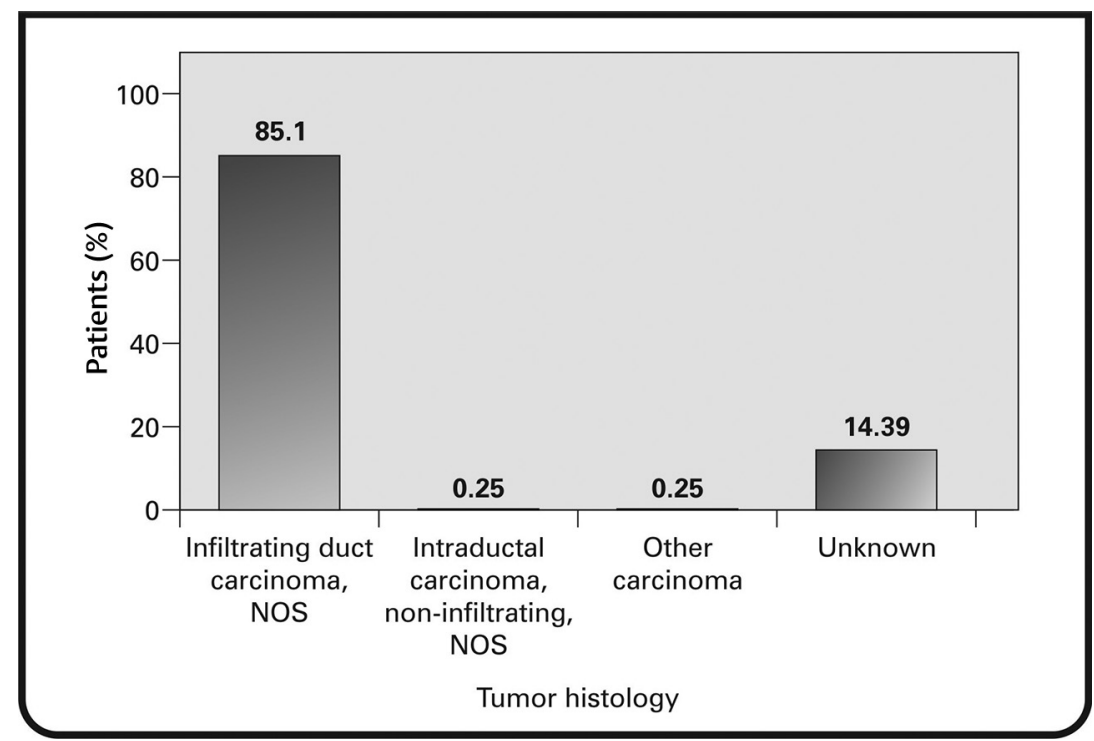

Fig. 7 Distribution of histological types of breast cancer in the study. NOS, not otherwise specified.

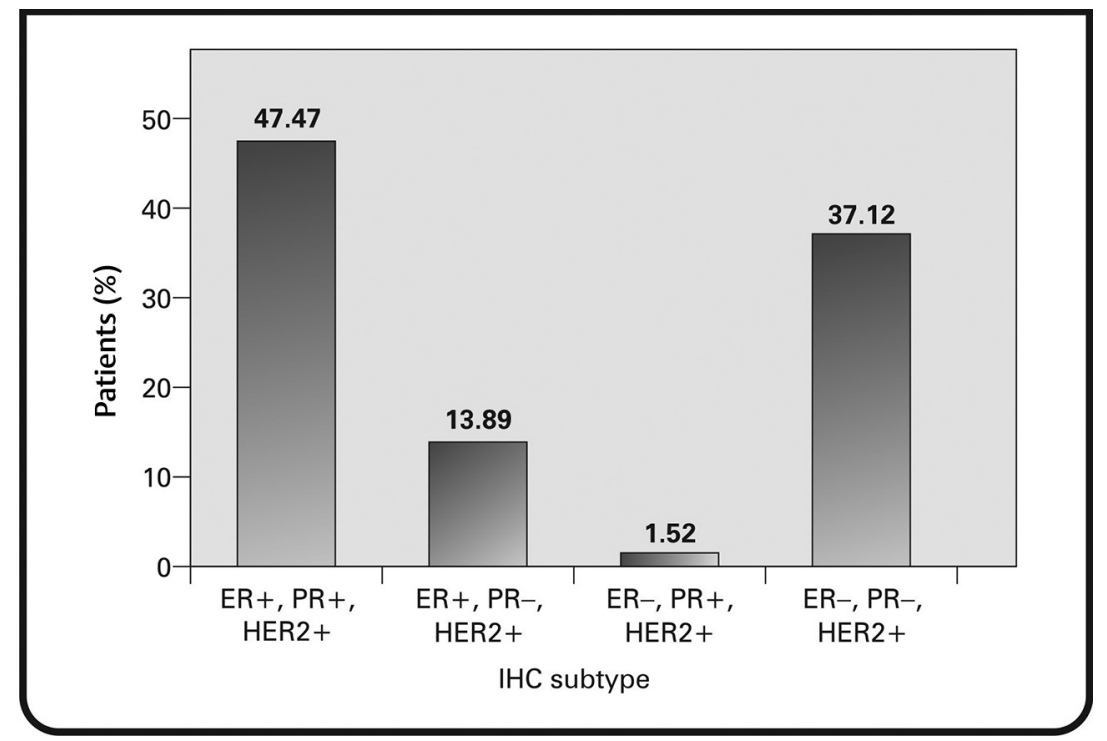

Fig. 8 Immunohistological subtypes distribution of tumors. ER, estrogen receptor; HER2+, human epidermal growth factor receptor 2+; IHC, immunohistochemistry; PR, progesterone receptor.

in the United Kingdom reported a significant increase in the breast cancer risk in patients with family history. Women who had two or more relatives with breast cancer had a 2.5 -fold increased risk of developing breast cancer. ${ }^{30}$ In our study, only $18.94 \%$ of patients reported a family history of breast cancer and $58.06 \%$ did not report any occurrence of breast cancer among family members. Although a small percentage of our study cohort displayed a familial relationship of breast cancer, family history cannot be ruled out as a determining risk factor.

To assess the HER2 status, we used IHC in $97.73 \%$ of patients and ISH criteria in $92.68 \%$ of patients. It was observed that $21.96 \%$ of tumors assessed by IHC were borderline HER2 and 76.23\% were HER2-positive. Similarly, assessment by ISH revealed $97.02 \%$ of tumors to be HER2-positive and $1.63 \%$ to be equivocal for HER2. The proportion of patients with $\mathrm{ER}+\mathrm{PR}+\mathrm{HER} 2+$ tumors was greater (47.47\%), followed by
ER-PR-HER2+ tumors (37.12\%). In a study of 2,544 invasive breast cancer cases, 189 triple-negative cases were reported, in which Asians had the least prevalence (12 [6.3\%]). ${ }^{31}$ In an analysis of 36,810 patients with known HR+/HER2-status, the findings suggested that patients of Asian ethnicity were less likely to be diagnosed with triple-negative tumors. ${ }^{32}$ Furthermore, in a retrospective study by Tan et al, in 996 patients with breast cancer in Malaysia (30.3\% with HER2 overexpressing tumors), the HER2 overexpressing subtype was noted to be more aggressive, with a significant relationship with PR-negativity. ${ }^{18}$

In our study, the staging and grading of the tumors were performed at diagnosis. We observed that more than half (51.01\%) of the patients in the cohort were with late-stage cancer (stage 3 and stage 4). This finding is in agreement with previous studies, which have reported that most breast 
cancers are typically diagnosed in advanced stages, especially in low- and middle-income countries of Asia, in contrast to Western countries or developed nations in Asia. ${ }^{23,28}$ In Kuala Lumpur, $27 \%$ of cases were diagnosed at advanced stages. A similar finding was reported from Kota Kinabalu, Sarawak, where $52 \%$ were diagnosed at advanced stages. ${ }^{23}$ A retrospective analysis of breast cancer cases in the Central African Republic reported similar results: a high proportion (53.7\%) of tumors was found to be in stage $3 .{ }^{33}$ In India, a similar proportion (57\%) of cases was diagnosed at stages 3 and $4 .{ }^{34}$ Several factors are responsible for this trend in the less-developed countries of Asia: poverty, ignorance, fear, and economic factors being the most important. In Asian countries, most women play a subservient role, and the decision to identify and treat breast cancer lies in the hands of the spouse or family elders. Stigmatization is another major factor for nondisclosure of breast lumps by women. Hence, painless lumps may remain undiagnosed until the advanced stages, when ulceration and pain become pronounced. ${ }^{23}$ Furthermore, in Malaysia, fatalistic attitude, poor knowledge, and recognition of symptoms, as well as poor decision-making skills, have also been found to contribute significantly to the delayed presentation of breast cancer cases. ${ }^{23}$ Recently a shift in the diagnosis is being observed in Asia, due to improvements in the socioeconomic status of less-developed countries, enabling better education and access to health care. For example, it was observed in Shanghai, China, that the percentage of detection of breast cancer tumors at early stages $(0-2)$ has increased from 78.6 to $93.3 \%{ }^{28}$ Thus, regional, ethnic, and socioeconomic disparities are key drivers in late presentation of breast cancer in the Asia-Pacific region.

The TNM classification allows categorization of tumors based on the degree of local, regional, and the general extension of disease at the time of primary treatment, thus providing an objective and permanent description of the tumors. Tumor size and the extent of lymph node involvement are two key prognostic indicators in breast cancer. We observed in our study that about half the patients (47.98\%) presented tumors in the T2 stage, followed by T1 (22.7\%). A majority (27.53\%) had 1 to 3 positive lymph node involvement and $12.3 \%$ showed metastasis. Our results are congruent with the regional data, which suggest that Malay patients are at an increased risk of axillary lymph node involvement and metastasis for tumor sizes below $20 \mathrm{~mm}$ and 20 to $50 \mathrm{~mm} .{ }^{35}$ Similar findings have been reported in studies on breast cancer in Western countries. In an analysis of 72,000 women with invasive breast cancer in Germany, it was reported that $30 \%$ of patients presented with tumors in the T2 stage and that $~ 9 \%$ were stages T3 and T4. ${ }^{36} \mathrm{Sim}-$ ilarly, an evaluation of 24,740 breast cancer cases from the Surveillance, Epidemiology, and End Results program, United States, has revealed that the survival rate was $45.5 \%$ in cases with tumor diameter $>5 \mathrm{~cm}$ and positive lymph node involvement, whereas the survival rate in tumors $<2 \mathrm{~cm}$ with no node involvement was $96.3 \%{ }^{37}$ Another survival analysis of 1,894 patients in Canada showed similar results: the survival rate was $92 \%$ for small node-negative cancers and $80 \%$ for small node-positive cancers. ${ }^{38}$ As tumor size increases, survival decreases, irrespective of lymph node involvement. . $^{37,38}$ Thus, a linear relation can be found between tumor diameter/lymph node involvement and survival rate. A study conducted to evaluate the association between clinicopathological features and HER2 overexpression in breast cancer patients in Malaysia revealed that HER2 overexpression is significantly associated with higher tumor grade, and lymph node positivity and an aggressive disease pattern. ${ }^{18}$

In our study, $\sim 58.84 \%$ were stratified as "high-risk" patients, and $34.34 \%$ as "intermediate-risk" patients. The criteria used for risk stratification in our study have been validated and used in earlier studies. ${ }^{24,25}$ The high percentage of patients with intermediate-to-high risk may have been due to the aggressive tumor characteristics, including higher tumor stage/size, lymph node involvement, and vessel invasion. All these characteristics have been significantly associated with aggressive disease and reduced survival in HER2 overexpressing tumors. ${ }^{16,18}$

We identified that $85.35 \%$ of tumors in our cohort were infiltrating ductal carcinomas on histological analysis. Our finding is aligned with previously and currently reported trends of invasive ductal carcinoma. Ductal carcinomas are the most common histological subtype, accounting for 70 to $80 \%$ of breast cancers. ${ }^{39} \mathrm{~A}$ population-based study of 6,247 breast cancer cases revealed that $71.5 \%$ were invasive ductal carcinomas, most occurring in women aged 50 years and above. ${ }^{39}$ A retrospective study of $1,564,080$ women screened for breast cancer by mammography revealed that $78.8 \%$ had invasive cancer, of which $14.6 \%$ were ductal carcinoma. ${ }^{40}$ The mean age of detection was 56.7 years. The findings from the above studies strongly co-related with our findings, as a majority of our cohort had a mean age of 51 years and ductal carcinoma dominated the histological subtype.

Despite our interesting findings, our study had certain limitations. The number of patients analyzed fell short of the target number specified in the study protocol. Additionally, the proportion of HER2-positive breast cancer patients at each study center was not uniform.

In conclusion, interesting findings were elucidated from our study, the most significant being an increasing proportion of HER2+ tumors, particularly ER+, PR+, HER2+ and ER-, PR-HER2+ types. Most patients in our study presented with late-stage tumors; this could have been due to several factors, such as fear, ignorance, socioeconomic factors, ethnicity, use of alternative medicine, and health care deficiencies. The percentage of patients presenting with intermediateand high-risk HER2 overexpressing tumors, with invasive nature, large tumor size, high tumor grade, and lymph node involvement, was also high. These findings point to a clear unmet need of early screening and detection of HER2+ breast cancer cases in Malaysia. Therefore, the need of the hour is to emphasize on the early diagnosis and timely treatment of breast cancer patients with the aggressive HER2 overexpressing type. This can be achieved by strongly improving the awareness of breast cancer identification and reporting, among the general population. Furthermore, providing 
culturally-sensitive information and education material to improvise decision making may also be useful. Local health authorities should facilitate and support health centers with adequate training and equipment for screening of breast cancer cases. Both opportunistic and population-based screening and outreach programs may help enhance the awareness and uptake of breast cancer screening in this region. Furthermore, future studies analyzing the survival rate and progression-free survival of the treatments for HER2+ breast cancer may help provide a larger picture on the outcomes of treatment of these patients in Malaysia.

\section{Funding}

Roche (Malaysia) Sdn. Bhd. provided grants for this study.

\section{Conflict of Interest}

G.K. is an employee of Roche (Malaysia) Sdn. Bhd. The other authors have no conflict of interest.

\section{Acknowledgments}

We would like to thank BioQuest Solutions for providing writing assistance in this project.

\section{References}

1 FerlayJ, Soerjomataram I, Dikshit R, et al. Cancer incidence and mortality worldwide: sources, methods and major patterns in GLOBOCAN 2012. Int J Cancer 2015;136(5):E359-E386

2 Youlden DR, Cramb SM, Yip CH, Baade PD. Incidence and mortality of female breast cancer in the Asia-Pacific region. Cancer Biol Med 2014;11(2):101-115

3 Azizah AM, Ibrahim NS, Abdullah NH. Malaysian National Cancer Registry Report 2007-2011. Ministry of Health Malaysia. 2015. Available at: https://www.crc.gov.my/wpcontent/uploads/documents/report/MNCRRrepor2007-2011. pdf. Accessed October 15, 2018

4 Azizah AM, Devraj T, Bina RS, et al. Penang Cancer Registry Report 2004-2008. Penang State Health Department. 2015. Available at: http://www.ncsmpenang.org/storage/upload/ ncsm/files/PCR20042008(1).pdf. Accessed October 15, 2018

5 Razif SM, Sulaiman S, Hanie SS, et al. The contribution of reproductive factors and family history towards premenopausal breast cancer risk in Kuala Lumpur, Malaysia. Med J Malaysia 2011;66(3):220-226

6 Matalqah L, Radaideh K, Yusoff ZM, Awaisu A. Predictors of breast cancer among women in a northern state of Malaysia: a matched case-control study. Asian Pac J Cancer Prev 2011;12(6):1549-1553

7 Yip CH, bt Mohd Taib NA, Lau PC. Does a positive family history influence the presentation of breast cancer? Asian Pac J Cancer Prev 2008;9(1):63-65

8 Norsa'adah B, Rampal KG, Rahmah MA, Naing NN, Biswal BM. Diagnosis delay of breast cancer and its associated factors in Malaysian women. BMC Cancer 2011;11:141

9 Kamarudin R, Shah SA, Hidayah N. Lifestyle factors and breast cancer: a case-control study in Kuala Lumpur, Malaysia. Asian Pac J Cancer Prev 2006;7(1):51-54

10 Rejali L, Jaafar MH, Ismail NH. Serum selenium level and other risk factors for breast cancer among patients in a Malaysian hospital. Environ Health Prev Med 2007;12(3):105-110

11 Yu FQ, Murugiah MK, Khan AH, Mehmood T. Meta-synthesis exploring barriers to health seeking behaviour among Malaysian breast cancer patients. Asian Pac J Cancer Prev 2015;16(1):145-152
12 Khan TM, Leong JPY, Ming LC, Khan AH. Association of knowledge and cultural perceptions of Malaysian women with delay in diagnosis and treatment of breast cancer: a systematic review. Asian Pac J Cancer Prev 2015;16(13):5349-5357

13 Saxena N, Hartman M, Bhoo-Pathy N, et al. Breast cancer in South East Asia: comparison of presentation and outcome between a middle income and a high income country. World J Surg 2012;36(12):2838-2846

14 Yip CH, Taib NA, Mohamed I. Epidemiology of breast cancer in Malaysia. Asian Pac J Cancer Prev 2006;7(3):369-374

15 Eroles P, Bosch A, Pérez-Fidalgo JA, Lluch A. Molecular biology in breast cancer: intrinsic subtypes and signaling pathways. Cancer Treat Rev 2012;38(6):698-707

16 Spitale A, Mazzola P, Soldini D, Mazzucchelli L, Bordoni A. Breast cancer classification according to immunohistochemical markers: clinicopathologic features and short-term survival analysis in a population-based study from the South of Switzerland. Ann Oncol 2009;20(4):628-635

17 Pathmanathan N, Geng JS, Li W, et al. Human epidermal growth factor receptor 2 status of breast cancer patients in Asia: results from a large, multicountry study. Asia Pac J Clin Oncol 2016;12(4):369-379

18 Tan G-H, Choo W-Y, Taib NA, Yip CH. Factors associated with HER2 overexpression in breast cancer: experience in an Asian developing country. Asian Pac J Cancer Prev 2009;10(5):837-840

19 Robinson A, Cain H, Amonkar S, et al. Epidermal growth factor receptor (EGFR) expression confers poor prognosis in HER2 positive breast cancers. Eur J Surg Oncol 2013;39:515

20 Press MF, Pike MC, Chazin VR, et al. Her-2/neu expression in node-negative breast cancer: direct tissue quantitation by computerized image analysis and association of overexpression with increased risk of recurrent disease. Cancer Res 1993;53(20):4960-4970

21 Devi CRB, Tang TS, Corbex M. Incidence and risk factors for breast cancer subtypes in three distinct South-East Asian ethnic groups: Chinese, Malay and natives of Sarawak, Malaysia. Int J Cancer 2012;131(12):2869-2877

22 Best practice guidelines for quality human epidermal growth factor receptor 2 (HER-2) testing in breast cancer for HER2 targeted therapy in Malaysia. Available at: www.acadmed. org.my/. Accessed: February 15, 2019

23 Yip CH, Bhoo Pathy N, Teo SH. A review of breast cancer research in Malaysia. Med J Malaysia 2014;69(Suppl A) :8-22

24 Goldhirsch A, Wood WC, Gelber RD, Coates AS, Thürlimann B, Senn HJ; 10th St. Gallen conference. Progress and promise: highlights of the international expert consensus on the primary therapy of early breast cancer 2007. Ann Oncol 2007;18(7):1133-1144

25 Peiris H, Mudduwa L, Thalagala N, Jayatilake K. Validity of St Gallen risk categories in prognostication of breast cancer patients in Southern Sri Lanka. BMC Womens Health 2018;18(1):30

26 Yersal O, Barutca S. Biological subtypes of breast cancer: prognostic and therapeutic implications. World J Clin Oncol 2014;5(3):412-424

27 Tan MM, Ho WK, Yoon SY, et al. A case-control study of breast cancer risk factors in 7,663 women in Malaysia. PLoS One 2018;13(9):e0203469

28 Fan L, Goss PE, Strasser-Weippl K. Current status and future projections of breast cancer in Asia. Breast Care (Basel) 2015; 10(6):372-378

29 Braithwaite D, Miglioretti DL, Zhu W, et al; Breast Cancer Surveillance Consortium. Family history and breast cancer risk among older women in the breast cancer surveillance consortium cohort. JAMA Intern Med 2018;178(4):494-501

30 Brewer HR, Jones ME, Schoemaker MJ, Ashworth A, Swerdlow AJ. Family history and risk of breast cancer: an 
analysis accounting for family structure. Breast Cancer Res Treat 2017;165(1):193-200

31 Kwan ML, Kushi LH, Weltzien E, et al. Epidemiology of breast cancer subtypes in two prospective cohort studies of breast cancer survivors. Breast Cancer Res 2009;11(3):R31

32 Howlader N, Altekruse SF, Li CI, et al. US incidence of breast cancer subtypes defined by joint hormone receptor and HER2 status. J Natl Cancer Inst 2014;106(5):1-8

33 Balekouzou A, Yin P, Pamatika CM, et al. Epidemiology of breast cancer: retrospective study in the Central African Republic. BMC Public Health 2016;16(1):1230

34 Hebert JR, Ghumare SS, Gupta PC. Stage at diagnosis and relative differences in breast and prostate cancer incidence in India: comparison with the United States. Asian Pac J Cancer Prev 2006;7(4):547-555

35 Bhoo-Pathy N, Hartman M, Yip CH, et al. Ethnic differences in survival after breast cancer in South East Asia. PLoS One 2012;7(2):e30995
36 Eisemann N, Waldmann A, Katalinic A. Epidemiology of breast cancer - current figures and trends. Geburtshilfe Frauenheilkd 2013;73(2):130-135

37 Carter CL, Allen C, Henson DE. Relation of tumor size, lymph node status, and survival in 24,740 breast cancer cases. Cancer 1989;63(1):181-187

38 Narod SA. Tumour size predicts long-term survival among women with lymph node-positive breast cancer. Curr Oncol 2012;19(5):249-253

39 Verkooijen HM, Fioretta G, Vlastos G, et al. Important increase of invasive lobular breast cancer incidence in Geneva, Switzerland. Int J Cancer 2003;104(6):778-781

40 Román M, Rué M, Sala M, et al; Cumulative False Positive Risk Group. Trends in detection of invasive cancer and ductal carcinoma in situ at biennial screening mammography in Spain: a retrospective cohort study. PLoS One 2013;8(12):e83121 